\title{
LASIK as an alternative line to treat noncompliant esotropic children
}

This article was published in the following Dove Press journal:

Clinical Ophthalmology

19 December 2011

Number of times this article has been viewed

\section{Ahmed M Saeed Mohamed A Abdrabbo \\ Ebsar Eye Center, Benha University, Benha, Egypt}

Purpose: To assess the safety and efficacy of laser-assisted in situ keratomileusis (LASIK) in facilitating strabismus management in noncompliant children with fully accommodative esotropia.

Setting: Ebsar Eye Center, Benha University (Benha, Egypt).

Methods: The study comprised 20 eyes of ten consecutive patients with accommodative esotropia. All patients were noncompliant with glasses and had refractive stability. They received brief general anesthesia and had bilateral LASIK using Wavelight ${ }^{\circledR}$ Algretto Wave ${ }^{\circledR}$ Eye-Q Excimer Laser (Alcon, Inc, Hunenberg, Switzerland) to fully correct their hyperopic refractive errors aiming to achieve orthophoria. Preoperative and postoperative best corrected visual acuity, cycloplegic refraction, angle of squint, and any LASIK complications were recorded. Follow-up period was 9 months.

Results: The age of patients ranged 5.1-9.2 years and the hyperopic error range was $+3.5 \mathrm{D}$ to $+6.75 \mathrm{D}$, with anisometropia $2 \mathrm{D}$ or less. No patient had decreased best corrected visual acuity or loss of fusion ability. The postoperative refractive error ranged from $-0.75 \mathrm{D}$ to $+1.5 \mathrm{D}$ at the end of the study period. All patients achieved orthophoria. No significant intraoperative or postoperative complications were recorded.

Conclusion: LASIK appears to be effective and relatively safe to treat accommodative esotropic children by reducing their hyperopic refractive error, however, patient selection is critical. Larger studies with longer follow-up are necessary to determine its long-term effects.

Keywords: accommodative esotropia, hyperopia, keratorefractive surgery, refractive error, esodeviation

\section{Introduction}

Accommodative esotropia is defined as a convergent ocular deviation associated with activation of the near synkinetic reflex to eliminate blurred retinal images produced by high hyperopic refractive errors. ${ }^{1}$ Accommodation can lead to excessive convergence, which eventually exceeds the fusional divergence amplitude, resulting in esotropia. Refractive accommodative esotropia is characterized by high hyperopia (mean +4.75 diopters [D]; range $+3.00 \mathrm{D}$ to $+10.00 \mathrm{D})$, moderate angle of esodeviation $(20-30$ prism diopters $[\mathrm{PD}])$, and normal accommodative convergence to accommodation ratio (difference of $10 \mathrm{PD}$ or less between distance and near esodeviation). ${ }^{2}$ It is the most common type of strabismus, with very favorable prognosis if the appropriate treatment is initiated promptly. ${ }^{3}$

Traditional methods of treating accommodative or partially accommodative esotropia include spectacle or contact lens correction of the hyperopic refractive error determined under full cycloplegia. ${ }^{4-6}$
Zayed Tower, El-souq Eltougary St,

El-Qanatir El-Khairiya,

Al-Qalyubiyah, Egypt

Email a_saeed775@yahoo.com 
Spectacles usually carry a psychological burden to the child and his family especially the females, limiting his activities, and cause a cosmetic problem, particularly in underdeveloped communities with low cultural and educational levels. It was found that a considerable proportion of these fully accommodative children who had their vision corrected by spectacles, get rid of their glasses and lose their follow-up visits. This noncompliance leads to loss of stereopsis and development of ammetropic and strabismic amblyopia which is too hard to correct later on. ${ }^{7}$ Contact lenses are also not a suitable line of treatment for these children as they carry a risk of many complications, especially allergic and infectious keratoconjunctivitis.

Recently, there have been several reports demonstrating the efficacy and safety of keratorefractive surgery for correction of refractive errors and esodeviation in patients with refractive accommodative esotropia. ${ }^{8-11}$

Laser-assisted in situ keratomileusis (LASIK) and photorefractive keratectomy (PRK) are the most commonly used keratorefractive procedures. Although PRK theoretically is safer as it avoids flap-related complications, LASIK has the ability to correct larger refractive errors, better predictability, faster healing times, less postoperative discomfort, less haze, shorter course of postoperative steroids, and avoidance of postoperative contact lens use for long time and preservation of Bowman's membrane. ${ }^{8,10,11}$

As ocular alignment is a matter of concern, it is important to consider amblyopia and the fusional status of the eyes prior to keratorefractive surgery. ${ }^{12-14}$

\section{Patients and methods}

The study comprised 20 eyes of ten consecutive pediatric patients with fully accommodative esotropia (refractive esotropia). The patients were selected from the outpatient clinic of Benha University Hospital (Benha, Egypt). The study was applied in the period from January 2010 to November 2010. The age of patients ranged 5.1-9.2 years. The mean age was 7.07 years with standard deviation of 1.46 . Six patients were males and four patients were females.

All patients were noncompliant with glasses (the children were urged to continuously wear their spectacles at every visit over a period of at least 12 months) and had refractive stability over the same period (cycloplegic refractive error was within $\pm 0.5 \mathrm{D}$ in at least three measurements taken 6 months apart).

Exclusion criteria included: any other ocular disease (other than refractive errors or esotropia), oblique muscle dysfunction, previous medical or surgical treatment for strabismus, family history of keratoconus or any corneal disease, failure to follow-up, and failure to get written consent. Informed consent was obtained from the children's parents acknowledging that the intended result of treatment was to correct both hyperopia and esotropia. They were informed that they were among the first patients to undergo refractive surgery for the correction of strabismus, and that no long-term outcome data for such treatment were available.

The preoperative evaluation included:

- Full cycloplegic refraction to determine the refractive error; total hyperopia was determined by instilling three drops of cyclopentolate 1.0\% (Cycloplejico ${ }^{\circledR}$ eye drop; Behestan Distribution Company, Tehran, Iran) 20 minutes apart and performing autorefractometry 30 minutes after instillation of the third drop.

- The patient's uncorrected visual acuity (UCVA) and best corrected visual acuity (BCVA) was evaluated (in decimal notation) using Snellen charts.

- The strabismic angle was evaluated using the prism and alternate cover test by measuring the amount of esotropia present at distance $(6 \mathrm{~m})$ and at near $(33 \mathrm{~cm})$ with and without correction (in PD). All patients were orthophoric with correction in place. All patients' distance and near deviations were the same (purely refractive accommodative esotropia).

- Accommodative convergence to accommodation ratios were measured by the gradient method.

- Stereopsis was screened using horizontal Lang two-pencil test (to confirm the child's cooperation) and measured with Titmus stereoacuity test with glasses.

- Extraocular movements to ensure that eye movements were full.

- Complete ocular examination was performed:

- The anterior segment to assess the cornea, anterior chamber, and lens.

- The fundus with both direct and indirect ophthalmoscopes to note the appearance of the macula and optic nerve.

- Intraocular pressure (IOP) measurement.

- Corneal topography and pachymetry.

All patients underwent LASIK by the $200 \mathrm{~Hz}$ Wavelight ${ }^{\circledR}$ Algretto Wave ${ }^{\circledR}$ Eye-Q Excimer Laser (Alcon, Inc, Hunenberg, Switzerland). One surgeon performed all surgeries. The instruments and laser parameters were the same as those used for correction of hyperopia in adult patients without accommodative esotropia, to fully correct their cycloplegic hyperopic refractive error. ${ }^{8,9,11}$ 
All patients underwent bilateral simultaneous procedures with the use of brief intravenous general anesthesia (ketamine 1.5-2 mg/kg [Sigma Tech Pharmaceutical Ind, Quesna, Egypt], propofol $1 \mathrm{mg} / \mathrm{kg}$ [Fresenius Kabi Austria, Graz, Austria], atropine sulfate $0.01 \mathrm{mg} / \mathrm{kg}$ [El-Nile for Misr Pharmaceuticals, Cairo, Egypt]). Suction ring was applied at a low vacuum level to hold the eye fixed and well-centered after iris recognition by the tracker during laser treatment. After flap reposition, soft 1-day contact lenses (Focus Dailies, Ciba Vision, Aschaffenburg, Germany) were inserted and ocular shields were used to protect the eye until the patient gained full consciousness.

Postoperatively, patients instilled tobramycin $0.3 \%$ and dexamethasone $0.1 \%$ combination (Tobradex, Alcon, Puurs, Belgium) and artificial tears (sodium hyaluronate) eye drops (Hyfresh, Jamjoom Pharma, Jeddah, Saudi Arabia) every 2 hours for 2 days, and then decreased the frequency gradually over 1 week. Then, fluorometholone $0.1 \%$ eye drops (Flucon, Alcon, Puurs, Belgium) were instilled three times a day and were continued for 2 weeks, with the dosage titrated based on return of BCVA to preoperative baseline and IOP level. Artificial tears were prescribed for use three times a day for 1 month. Any intraoperative or postoperative LASIK complications were recorded.

Postoperative examination was carried out on the second day to check the flap and to remove the contact lenses, at 1 week to measure IOP and taper the drops, and at 1 month, 4 months, and 9 months to measure UCVA and BCVA, cycloplegic refraction, angle of squint, stereoacuity, and IOP.

\section{Statistical analysis}

The collected data were tabulated and analyzed using SPSS (v 17; SPSS, Inc, Chicago, IL). Software categorical data were presented as number and percentages while quantitative data were expressed as mean, standard deviation, and standard error of the mean or median and range. Paired $t$-test and Friedman test were used as tests of significance for comparing preoperative and postoperative data of the study group. Correlation coefficient ( $r$ ) was used to assess linear association between two measures and its values were used to calculate the mean regression percentage $\left(\mathrm{P}_{\mathrm{rm}}=100[1-r]\right)$. The accepted level of significance in this work was stated at 0.05 ( $P<0.05$ was considered significant).

\section{Results}

\section{Refractive outcomes}

The median spherical equivalent was $+4.88 \mathrm{D}$ (range +3.5 to +6.75 ) preoperatively, $+0.63 \mathrm{D}$ (range -0.75 to +1.5 ) at

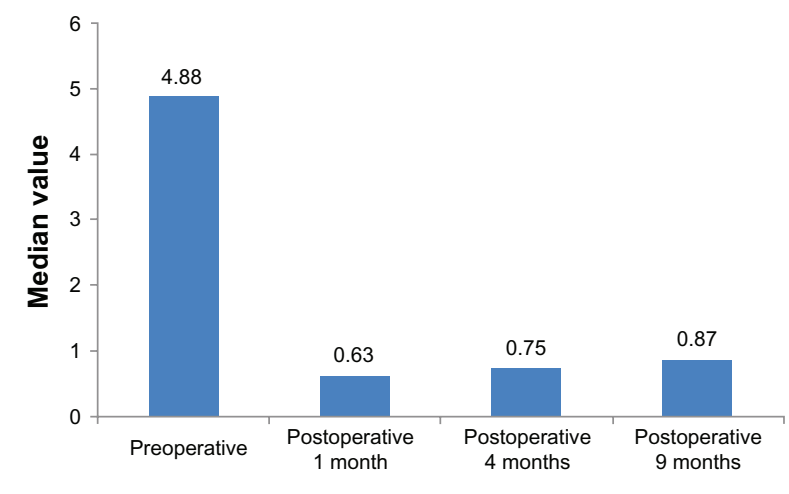

Figure I Comparison between the preoperative and postoperative spherical equivalent of the refractive error among the studied group.

1-month follow-up, $+0.75 \mathrm{D}$ (range -0.5 to +1.5 ) at 4-month follow-up, and $+0.87 \mathrm{D}$ (range -0.5 to +1.5 ) at final (9-month) follow-up. All eyes were within a range of $-0.5 \mathrm{D}$ to +1.5 D of emmetropia at final follow-up (Figure 1). There was a statistically significant improvement and correction of the refractive error $(P<0.001)$. No patients required retreatment.

A mean hyperopic change of $0.12 \mathrm{D}$ was observed between 1-month and 4-month follow-up, and between 4-month and 9-month follow-up (Table 1). This was not statistically significant. No enhancements were required or performed. No patients required glasses or prolonged contact lenses postoperatively (Figure 2).

\section{Visual acuity outcomes}

There was a statistically significant improvement of BCVA when comparing mean preoperative values $(0.51 \pm 0.16)$ with postoperative values at 4 months $(0.56 \pm 0.18)$ and 9 months $(0.6 \pm 0.17)(P=0.021$ and $<0.001$, respectively), but not at 1 month $(0.51 \pm 0.18, P=0.97)$ (Figure 3).

Mean preoperative BCVA was $0.51 \pm 0.16$ in decimal notation (range $0.2-0.8$ ). This relatively low value was due to the presence of three cases of hyperopic ammetropic amblyopia and two cases of minimal anisohyperopia. Mean BCVA at 1-month postoperative follow-up was $0.51 \pm 0.18$, which does

Table I Mean hypermetropic regression in the studied group

\begin{tabular}{|c|c|c|c|c|}
\hline & Mean & SD & $r$ & $P_{r m}=100(I-r)$ \\
\hline Preoperative SE of RE (D) & 5.08 & 1.01 & - & - \\
\hline Postoperative & 0.61 & 0.46 & 0.467 & $53.3 \%$ \\
\hline \multicolumn{5}{|l|}{ SE of RE I month (D) } \\
\hline Postoperative & 0.66 & 0.57 & 0.369 & $63.1 \%$ \\
\hline \multicolumn{5}{|l|}{ SE of RE 4 month (D) } \\
\hline Postoperative & 0.68 & 0.60 & 0.175 & $82.5 \%$ \\
\hline SE of RE 9 month (D) & & & & \\
\hline
\end{tabular}

Abbreviations: $\mathrm{D}$, diopter; $\mathrm{P}_{r m}$, percent of regression to the mean; $r$, correlation coefficient; RE, refractive error; SD, standard deviation; SE, spherical equivalent. 


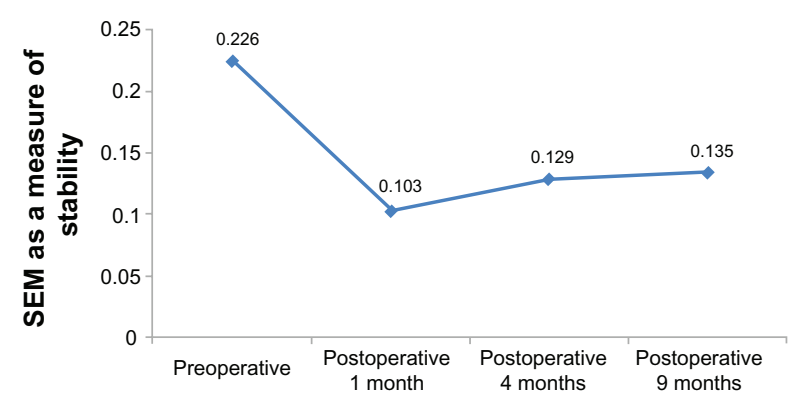

Figure 2 Standard error of the mean (SEM) as a measure of refractive stability in the study group.

not represent a statistically significant reduction in visual acuity $(P=0.97)$. A decrease in BCVA was observed at 1 -month follow-up in six eyes $(30 \%)$, an increase in ten eyes $(50 \%)$, and no difference in four eyes (20\%). During this interval, one patient had a reduction of three lines BCVA in one eye. These decreases in BCVA were attributed to temporary tear film instability, since no other plausible etiology could be identified. No patient had more than microstriae, and mild haze, when present, was located outside the visual axis. No other patient had a reduction of more than two lines BCVA at 1-month postoperative follow-up. Mean BCVA returned to the preoperative level ( 0.51 decimal notations) near the end of 1-month postoperative follow-up.

Mean preoperative UCVA was $0.29 \pm 0.11$ (range $0.1-$ 0.60). Mean postoperative UCVA was $0.55 \pm 0.17$ at 9 -month follow-up. This improvement in UCVA was statistically significant $(P=0.001)$, signifying a high refractive efficacy. UCVA improved in 17 eyes (85\%), was unchanged in two eyes $(10 \%)$, and decreased in one eye. There was no statistically significant change in mean UCVA at final follow-up when compared to mean UCVA at 1-month and 4-month follow-up ( $P=0.074$ and 0.065 , respectively); although there was a trend toward improvement, signifying refractive stability.

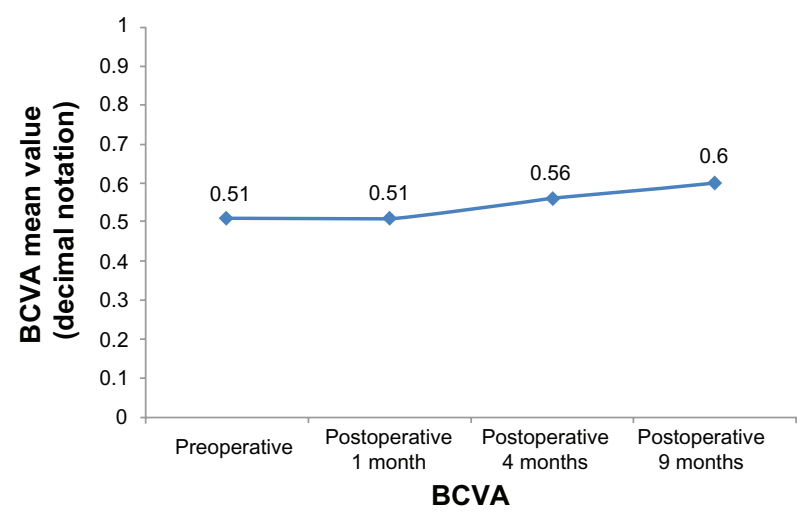

Figure 3 Preoperative and postoperative best corrected visual acuity (BCVA) values in the study group.

\section{Alignment outcomes}

Mean preoperative esotropic deviation at near and at distance without correction was 20.1 \pm 4.9 PD (range 14-30). Mean postoperative esotropic angle was $1.8 \pm 1.5 \mathrm{PD}$. This change was statistically significant $(P<0.001)$. Mean change was 18.3 PD. Near and distance angles were averaged for each participant before analysis $(n=10)$.

All patients had a normal accommodative convergence to accommodation ratio both preoperatively and postoperatively. All patients were orthotropic at distance and at near without correction at 1-month postoperative evaluation, and at all subsequent evaluations reported alignment stability.

\section{Sensory outcome}

The patients were examined using horizontal Lang two-pencil test to assess their cooperation in screening stereoacuity. All achieved positive results both preoperatively and postoperatively. Accurate measurement was done using Titmus stereoacuity test with glasses.

Mean preoperative stereoacuity (measured in seconds of arc) was $200 \pm 116.6$, which improved postoperatively to $176 \pm 117.6$ seconds of arc with no statistically significant value $(P=0.081)$. These relatively low values were due to the presence of three cases with ammetropic amblyopia and two cases with minimal anisometropia. Five patients $(50 \%)$ had no change in stereoacuity and five patients had an increase in stereoacuity (two patients gained 80 seconds of arc and three patients gained 40 seconds of arc), and no patient had a loss of stereoacuity after surgery. There was no change in stereoacuity between 1-month and final follow-up examination.

\section{IOP assessment}

Mean preoperative IOP was $13.7 \pm 1.7 \mathrm{mmHg}$. There was an increase in postoperative IOP at 1-month follow-up (mean IOP $13.9 \pm 5.6 \mathrm{mmHg})$, but not to a statistically significant level $(P=0.94)$. Mean postoperative IOP was $10.3 \pm 1.5 \mathrm{mmHg}$ at 4-month and 9-month follow-up, showing a statistically significant decrease in IOP $(P=0.001)$ when compared with preoperative values. Two patients had an elevated IOP (range 23-26 mmHg) during the first postoperative month that was controlled by withdrawal of topical steroids and instillation of topical antiglaucoma eye drops (timolol maleate $0.5 \%$ twice daily [Timolo, Eipico, 10th of Ramadan city, Egypt]). One patient had the same IOP, while the other seven patients had a decreased IOP at 1-month follow-up. All patients recorded a decreased IOP after the first postoperative month till the end of the study period. 


\section{Subjective outcomes}

All patients and their parents reported satisfaction with the functional and cosmetic results. Three patients (30\%) suffered from burning sensation, especially in the morning, due to dry eye that was relieved by frequent application of artificial tears. Two patients complained of blurred vision during the first postoperative week despite accurate correction and satisfactory visual acuity. One patient reported headache and eye ache for few days with unexplained cause.

\section{Complications}

There were no significant intraoperative or postoperative complications. There were no infections, flap-related complications, decentered ablations, or unexpected refractive outcomes. Microstriae, minimal haze, and tear film instability were seen in some patients, but resolved with postoperative medication regimen within the first postoperative month in all patients with corresponding return of BCVA to preoperative levels.

\section{Discussion}

Accommodative esotropia usually presents in preschool years, and it is the most common type of strabismus, accounting for approximately one half of all childhood esotropia. ${ }^{4}$ It is usually stable and does not resolve in young children with high hypermetropia especially without tight regular spectacle wear. The most important factor in predicting resolution of accommodative esotropia is the amount of hypermetropia at the time of diagnosis. In early-onset patients, incidence of high-level stereoacuity is much lower and incidence of deterioration of control requiring strabismus surgery is much higher. ${ }^{15}$ Thus, a young age group (5.1-9.2 years) was selected for this study.

The natural history of hypermetropia in accommodative esotropia is not clear; hypermetropia usually increases from initial examination to age 7 years, but by only a small amount. This may represent a true increase, or increased discovery of hypermetropia on subsequent cycloplegic refractions after a child wore glasses just from the relaxation of ciliary muscle tone. ${ }^{16}$ This emphasizes the importance of a follow-up cycloplegic refraction, and esotropia should only be considered purely accommodative until this follow-up.

Deviation is typically eliminated by controlling the accommodative effort with optical correction of hypermetropia. Full cycloplegic refraction is required to maintain adequate alignment and to achieve the best possible binocular vision result. Failure to fully correct hypermetropia in these situations leads to greater rates of distance-near disparity as well as to greater rates of deterioration; on the other hand, it may improve fusional vergence amplitude. ${ }^{15,16}$ So, full cycloplegic refraction was corrected after being stable for 12 months.

The stability of refractive error over a period of 1 year prior to the procedure (cycloplegic refractive error was within $\pm 0.5 \mathrm{D}$ ) was emphasized in the present study, as by Sabetti et al and Dvali et al in previous studies. ${ }^{17,18}$ An age group (5.1-9.2 years) younger than any other study was treated, encouraged by the full and stable cycloplegic refractive error.

Some rural areas in Egypt are of low cultural, educational, and social levels. Spectacles always carry a psychological burden to children of both sexes and their families in these areas. None of these children was developmentally delayed, but noncompliance with glasses was mainly due to the social barrier between the child and his peers. The parents refused spectacles - even when the strabismus was corrected - as this meant that their child was lacking vision and inferior to other children. When the need for spectacles was stressed as the only way to treat strabismus, as strabismus surgery has no role in this condition, the parents asked for LASIK to get rid of the glasses. Such cases were included in the study after detailed explanation and discussion of the benefits and drawbacks of the procedure.

Regression is common after hyperopic LASIK so long term follow-up is essential for these children; the parents were informed that over a few years, gradually increasing hyperopia might cause reoccurrence of the strabismus or require a return to wearing glasses to maintain control. Any significant error affecting either the orthotropic state or BCVA should be further corrected. Esotropes lose much of their error in the teenage years and myopic shift is expected to occur well past the age of the patients in this study. This was clearly explained to the child's family prior to the surgery. The aim was short-term correction of strabismus, improved visual acuity, and allowing better optokinetic and psychovisual development in this critical period of the child's life without any social or psychological barrier between the child and his/her peers - even if further intervention may be needed later in life if the refraction shifts towards myopia. Such intervention may be in the form of glasses, contact lenses, or even redoing LASIK, but this would be later after stabilizing BCVA and orthotropia and when they are older (adult or late teen). All families accepted this potential risk against the dependence of their children on glasses (a written consent was obtained).

Both PRK and LASIK have been used to treat refractive accommodative esotropia, but the patient characteristics and 
outcomes vary. ${ }^{10}$ Sabetti et $\mathrm{al}^{17}$ compared PRK and LASIK for the treatment of refractive accommodative esotropia in young adults and found no difference in the mean postoperative angle of esotropia between the two modalities, suggesting that LASIK was the preferred modality for larger magnitudes of hyperopia. Dvali et $\mathrm{al}^{18}$ advocated LASIK for school-aged children with severe anisometropia and high astigmatism. However, Hutchinson et al ${ }^{10}$ believed that for small to moderate hyperopic refractive errors $(<5 \mathrm{D})$ associated with refractive accommodative esotropia, PRK is predictable and safe. The extra measure of safety provided by PRK is being considered in school-aged children and young adults in whom the risks of flap complications and corneal ectasia are likely to be of greater concern. Hutchinson et $\mathrm{al}^{10}$ suggested that none of their patients had postoperative residual hyperopia, in contrast to many other studies, in which a substantial number of patients were left undercorrected or required retreatment. $8,9,19$

All patients underwent bilateral simultaneous procedures because this minimized the disruption of binocularity, consolidated the recovery period, decreased the risk of general anesthesia, and was preferred by the surgeon to a sequential procedure.

In agreement with Magli et al, ${ }^{11}$ who treated 17 patients of his study group with LASIK and three patients with PRK (range 14-24 years, mean 18.8 years), the present study found that LASIK was effective and safe in young or adolescent patients.

The mean hyperopic change was $0.07 \mathrm{D}$ between the 1-month and 9-month postoperative follow-up, which is much less than that reported by Farahi and Hashemi $(0.68 \mathrm{D})$ in their ten patients with fully and partially accommodative esotropia (ages 11-43 years) treated with LASIK. Their attempted correction was also full cycloplegic refraction $($ mean +5.03$) .{ }^{19}$

The intended goal of surgery was achieved in all patients as they were all orthotropic (postoperative uncorrected ocular alignment was the same as preoperative spectaclecorrected alignment). Although several studies showed that good alignment can be achieved even in patients with residual hyperopia, ${ }^{8,9,19}$ this differs from the results of Stidham et al ${ }^{20}$ who performed LASIK for 10 patients with purely refractive accommodative esotropia. Only two became orthophoric, four patients converted from an esotropia to an esophoria, and four showed no reduction in their deviation. This could be attributed to the high mean preoperative spherical equivalent (+7.36 D) with mean attempted undercorrection (6 D), postoperative residual spherical equivalent error averaged 2.1 D, and there were visually significant flap-related complications.
Interestingly, there were a number of patients in their study who were orthophoric postoperatively in spite of a residual hyperopic refractive error of over $2 \mathrm{D}$.

No patient in the present study required retreatment for residual hyperopia. This could be because the full cycloplegic refraction was corrected, with range of +3.5 to $+6.75 \mathrm{D}$, after 1 year of stable refraction and in an age group with mean 7 years (not younger). This is similar to Hutchinson et al, ${ }^{10}$ who suggested that none of their patients had postoperative residual hyperopia in a study using PRK to treat 40 patients with a mean spherical equivalent of $+3.06 \mathrm{D}$, and Magli et al, ${ }^{11}$ who also realized emmetropia $( \pm 1.00 \mathrm{D})$ in $97.5 \%$ of eyes $(39 / 40)$ with a mean correction of $+4.62 \mathrm{D}$ (range +2.25 to +7.75 , standard deviation 4.52 ) obtained with excimer laser.

This differs from the results of Phillips et $\mathrm{al}^{9}$ where retreatment was required due to undercorrection in $34 \% \pm 17 \%$ of their patients, which they attributed to the high variability inherent in hyperopic LASIK. They suggest that the development of a different nomogram might be beneficial. Also, Hoyos et $\mathrm{al}^{8}$ reported that half of the eyes required retreatment within 3-5 months of initial treatment.

Only minor changes in stereoacuity were seen after LASIK in the current study, with five patients having improvements in stereoacuity and five patients experiencing no change. These changes in stereoacuity were attributed to testing variability since no other plausible explanation could be identified and since an equal number of patients "gained" as "preserved" stereoacuity. The relatively low values together with nonimprovement could be explained by the preoperative findings of three cases with ammetropic amblyopia and two cases with minimal anisometropia. These results confirm the plan for simultaneous bilateral LASIK correction in the same sitting. This was also confirmed by Birch and Wang ${ }^{14}$ who stated that improved stereoacuity outcomes are associated with better long-term stability of alignment, reduced risk for and severity of amblyopia, and improved achievement of sensorimotor developmental milestones.

There were no significant complications reported in the current study, which was also reported by Phillips et $\mathrm{al}^{9}$ and Magli et al. ${ }^{11}$ This is in contrast to Hoyos et $\mathrm{al}^{8}$ who reported many complications in a nine-patient study including topographic decentration in three eyes, superficial punctate epitheliopathy in two eyes, and peripheral epithelialization in two eyes after retreatment. One patient with decentration complained of glare and night halos. Also, Stidham et $\mathrm{al}^{20}$ found visually significant flap striae in $25 \%$ of eyes, 
decentered ablations in $8 \%$ of eyes, diffuse lamellar keratitis was treated in $4 \%$ of eyes, and $23 \%$ of patients lost one or more lines of BCVA.

The current results confirm that LASIK can be used successfully to treat hyperopia and purely refractive accommodative esotropia in patients with hyperopia up to $+6.75 \mathrm{D}$. Although hyperopic regression was seen between the first postoperative month and 4-month and 9-month postoperative follow-up, it was very small and it was found that refractive error and motor alignment remained stable.

Two patients had an elevated IOP (range 23-26 $\mathrm{mmHg}$ ) during the first postoperative month due to moderate response to topical steroids. This IOP rise was controlled by steroid withdrawal and instillation of topical antiglaucoma eye drops. This should arouse attention as a large percentage of children show a steroid response that may lead to glaucoma and bad visual impact. Kwok et $\mathrm{a}^{21}$ found that $56 \%$ of children are high responders to topical dexamethasone. Thus, IOP was followed up throughout the study period.

Limitations of this study include its limited number, short follow-up period, nature, lack of comparison group, and limited generalizability due to the select patient population with a small age range and hypermetropic error.

In conclusion, LASIK appears to be effective and relatively safe to treat accommodative esotropia, even in young children, by reducing their hyperopic refractive error and eliminating the need for spectacle or contact lens correction. However, patient selection is critical, and longer follow-up is needed.

\section{Disclosure}

The authors report no conflicts of interest in this work.

\section{References}

1. Parks MM. Ocular motility and strabismus. Hagerstown, MA: Harper \& Row; 1975.

2. Parks MM. Abnormal accommodative convergence in squint. AMA Arch Ophthalmol. 1958;59(3):364-380.

3. Parks MM. Management of acquired esotropia. Br J Ophthalmol. 1974;58(3):240-247.

Clinical Ophthalmology

\section{Publish your work in this journal}

Clinical Ophthalmology is an international, peer-reviewed journal covering all subspecialties within ophthalmology. Key topics include: Optometry; Visual science; Pharmacology and drug therapy in eye diseases; Basic Sciences; Primary and Secondary eye care; Patient Safety and Quality of Care Improvements. This journal is indexed on
4. Mohney BG. Common forms of childhood esotropia. Ophthalmology. 2001;108(4):805-809.

5. Cho YA, Yi S, Kim SW. Clinical evaluation of cessation of hyperopia in 123 children with accommodative esotropia treated with glasses for best corrected vision. Acta Ophthalmol. 2009;87(5):532-537.

6. Li CH, Chen PL, Chen JT, Fu JJ. Different corrections of hypermetropic errors in the successful treatment of hypermetropic amblyopia in children 3 to 7 years of age. Am J Ophthalmol. 2009;147(2):357-363.

7. Rutstein RP. Update on accommodative esotropia. Optometry. 2008; 79(8):422-431.

8. Hoyos JE, Cigales M, Hoyos-Chacon J, Ferrer J, Maldonado-Bas A. Hyperopic laser in situ keratomileusis for refractive accommodative esotropia. J Cataract Refract Surg. 2002;28(9):1522-1529.

9. Phillips CB, Prager TC, McClellan G, Mintz-Hittner HA. Laser in situ keratomileusis for high hyperopia in awake, autofixating pediatric and adolescent patients with fully or partially accommodative esotropia. $J$ Cataract Refract Surg. 2004;30(10):2124-2129.

10. Hutchinson AK, Serafino M, Nucci P. Photorefractive keratectomy for the treatment of purely refractive accommodative esotropia: 6 years' experience. Br J Ophthalmol. 2010;94(2):236-240.

11. Magli A, Iovine A, Gagliardi V, Fimiani F, Nucci P. LASIK and PRK in refractive accommodative esotropia: a retrospective study on 20 adolescent and adult patients. Eur J Ophthalmol. 2009;19(2):188-195.

12. Parks MM, Mitchell PR, Wheeler MB. Concomitant esodeviations. In: Tasman W, Jaeger EA, editors. Duane's Clinical Ophthalmology. Philadelphia, PA: JB Lippincott; 1998:1-21.

13. Birch EE, Stager DR Sr, Berry P, Leffler J. Stereopsis and long-term stability of alignment in esotropia. J AAPOS. 2004;8(2):146-150.

14. Birch EE, Wang J. Stereoacuity outcomes after treatment of infantile and accommodative esotropia. Optom Vis Sci. 2009;86(6):647-652.

15. Black BC. The influence of refractive error management on the natural history and treatment outcome of accommodative esotropia (an American Ophthalmological Society thesis). Trans Am Ophthalmol Soc. 2006;104:303-321.

16. Rosenbaum AL, Bateman JB, Bremer DL, Liu PY. Cycloplegic refraction in esotropic children. Cyclopentolate versus atropine. Ophthalmology. 1981;88(10):1031-1034.

17. Sabetti L, Spadea L, D'Alessandri L, Balestrazzi E. Photorefractive keratectomy and laser in situ keratomileusis in refractive accommodative esotropia. J Cataract Refract Surg. 2005;31(10):1899-1903.

18. Dvali ML, Tsintsadze NA, Mirtskhulava SI. Features of hyperopic LASIK in children. J Refract Surg. 2005;21(Suppl 5):S614-S616.

19. Farahi A, Hashemi H. The effect of hyperopic laser in situ keratomileusis on refractive accommodative esotropia. Eur J Ophthalmol. 2005;15(6):688-694.

20. Stidham DB, Borissova O, Borissov V, Prager TC. Effect of hyperopic laser in situ keratomileusis on ocular alignment and stereopsis in patients with accommodative esotropia. Ophthalmology. 2002;109(6): $1148-1153$.

21. Kwok AK, Lam DS, Ng JS, Fan DS, Chew SJ, Tso MO. Ocular hypertensive response to topical steroid in children. Ophthalmology. 1997; 104(12):2112-2116. 